\title{
Maternal exposure to angiotensin converting enzyme inhibitors in the first trimester and risk of malformations in offspring: a retrospective cohort study (c) (1) (8) OPEN ACCESS
}

\author{
De-Kun Li principal investigator ${ }^{1}$, Chunmei Yang program analyst ${ }^{1}$, Susan Andrade research \\ associate professor ${ }^{2}$, Venessa Tavares program analyst ${ }^{1}$, Jeannette R Ferber program analyst ${ }^{1}$
}

${ }^{1}$ Division of Research, Kaiser Foundation Research Institute, Kaiser Permanente, Oakland, CA 94612, US; ${ }^{2}$ Meyers Primary Care Institute, Worcester, MA 01605, US

\begin{abstract}
Objective To examine a reported association between use of angiotensin converting enzyme (ACE) inhibitors during the first trimester and risk of malformations in offspring.

Design A population based, retrospective cohort study linking automated clinical and pharmacy databases including comprehensive electronic medical records.

Participants Pregnant women and their live born offspring (465 754 mother-infant pairs) in the Kaiser Permanente Northern California region from 1995 to 2008.

Main outcome measure Congenital malformation in live births.

Results The prevalence of ACE inhibitor use in the first trimester only was $0.9 / 1000$, and the use of other antihypertensive medications was 2.4/1000. After adjustment for maternal age, ethnicity, parity, and obesity, use of ACE inhibitors during the first trimester only seemed to be associated with increased risk of congenital heart defects in offspring compared with normal controls (those with neither hypertension nor use of any antihypertensives during pregnancy) (15/381 (3.9\%) $v$ $6232 / 400021$ (1.6\%) cases, odds ratio 1.54 (95\% confidence interval 0.90 to 2.62)). A similar association was observed for use of other antihypertensives (28/1090 (2.6\%) cases of congenital heart defects, odds ratio 1.52 (1.04 to 2.21)). However, compared with hypertension controls (those with a diagnosis of hypertension but without use of antihypertensives) (708/29 735 (2.4\%) cases of congenital heart defects), neither use of ACE inhibitors or of other antihypertensives in the first trimester was associated with increased congenital heart defects risk (odds ratios 1.14 (0.65 to 1.98 ) and1.12 (0.76 to 1.64 ) respectively).

Conclusions Maternal use of ACE inhibitors in the first trimester has a risk profile similar to the use of other antihypertensives regarding malformations in live born offspring. The apparent increased risk of
\end{abstract}

malformations associated with use of ACE inhibitors (and other antihypertensives) in the first trimester is likely due to the underlying hypertension rather than the medications.

\section{Introduction}

Angiotensin converting enzyme (ACE) inhibitors are a class of antihypertensive drug and are among the most widely prescribed drugs for hypertension. Their fetal toxicity in the second or third trimesters has been well documented,,${ }^{12}$ but, until recently, their teratogenic effect in the first trimester was largely unknown. ${ }^{34}$ A study based on data from the Tennessee Medicaid population reported an increased risk of congenital anomalies associated with use of ACE inhibitors during the first trimester, raising the possibility of a teratogenic effect. ${ }^{45}$ This association seemed unique to ACE inhibitors because there was no such association with use of other antihypertensives in the same study. ${ }^{5}$ However, two subsequent studies did not find the same pattern of association with only ACE inhibitor use: both reported an increased risk associated with use of ACE inhibitors during the first trimester, but use of other types of antihypertensive also showed an increased risk. ${ }^{67}$ These findings fail to confirm a specific effect with ACE inhibitors and raise the question of whether the observed risk associated with antihypertensives in general was due to the effect of the drugs or the underlying hypertension.

To examine the potential association between maternal use of ACE inhibitors during the first trimester and the risk of malformations in offspring, especially congenital heart defects and neural tube defects, we conducted a large, population based, cohort study based on information from more than 465000 mother-infant pairs from 1995 to 2008 in the Kaiser Permanente 
Northern California system. Our aim was to clarify whether the observed risk was unique to ACE inhibitor use, to antihypertensives in general, or to the underlying hypertension.

\section{Methods}

A population based, retrospective, cohort study was conducted among the Kaiser Permanente Northern California member population of women who delivered a live birth from 1995 to 2008. The study was approved by the Kaiser Permanente Northern California Institutional Review Board. Kaiser Permanente Northern California has well established automated clinical and pharmacy databases that capture delivery, diagnosis of malformation, dispenses of prescription drugs, and presence of hypertension during pregnancy. In addition, through linkage to various databases including California birth certificate data and the Kaiser Permanente Northern California

Alpha-Fetoprotein Prenatal Screening Program, we were able to obtain additional variables (such as maternal pre-existing diabetes, pregnancy weight, age, ethnicity, education) for adjustment as potential confounders. To examine the risk of malformation in offspring, we included all live births in the study population.

\section{Ascertainment of maternal exposure to ACE inhibitors and other antihypertensives during pregnancy}

The Pharmacy Information Management System is a computerised pharmacy prescription and dispensation database in Kaiser Permanente Northern California. This captures all prescription drugs dispensed, with information on date dispensed, dose, and days of supply. Use of ACE inhibitors and other antihypertensives during pregnancy was ascertained through linkage of the information from the database to women who delivered live births from 1 January 1995 to 31 December 2008 identified from the Kaiser Permanente Northern California clinical databases.

Last menstrual period was determined based on gestational age recorded in the Kaiser Permanente Northern California clinical databases in combination with the calendar date at delivery (last menstrual period=date at delivery-gestational age). Through linkage to information on dates of drug dispensation and days of supply from the pharmacy database, we were able to identify women who were exposed to ACE inhibitors and other antihypertensives during pregnancy. We classified the timing of exposure as: (a) use during the first trimester only, $(b)$ use during the second or third trimester only, and $(c)$ use at any time during pregnancy. Two cohorts of women who were exposed to antihypertensive drugs during pregnancy were established: a cohort exposed to ACE inhibitors and a cohort exposed to antihypertensives other than ACE inhibitors. Any pregnant women who used ACE inhibitors in isolation or in combination with other drugs were classified into the cohort exposed to ACE inhibitors. We excluded a few users of angiotensin receptor blockers in this cohort since they have some pharmacological similarities with ACE inhibitors. Users of all other antihypertensive drugs (see appendix on bmj.com for the list) were classified into the cohort of "other antihypertensive drug users."

\section{Comparison cohorts}

One of the most difficult methodological challenges in pharmacoepidemiological studies is to control for the underlying indication for the drugs being studied. Therefore, establishing a comparable control group is crucial. Since the underlying condition for ACE inhibitors is hypertension, we identified pregnant women who had a diagnosis of hypertension at any time from one year before their last menstrual period to the end of their pregnancy but who were not prescribed any antihypertensive drugs (based on our pharmacy data). This group served as a control for the underlying indication of antihypertensive drugs (that is, hypertension controls). The remaining pregnant women who had neither a diagnosis of hypertension nor use of any antihypertensive drug formed the normal control group.

\section{Ascertainment of malformations}

All live born infants in the identified eligible mother-infant pairs were linked to clinical databases (both inpatient and outpatient data) including electronic medical records to identify diagnoses of major malformations (ICD-9 CM (international classification of diseases, ninth revision) codes 740.0-759.9). In order to allow comparisons, we included malformations of similar types to those included in the study by Cooper et al that reported the association between ACE inhibitor use in the first trimester and malformation risk in offspring. ${ }^{5}$

All infants were followed to the end of the study period, 31 December 2008, to maximise the use of existing clinical information for a more complete ascertainment of malformations. However, most malformations (for example, $89 \%$ of congenital heart defects identified) were diagnosed in the infants before 1 year of age.

While the Kaiser Permanente Northern California automated databases have been shown to be comprehensive and accurate, ${ }^{89}$ we conducted a validation study of the diagnoses of malformations by comparing the diagnoses between medical charts and our clinical databases. We randomly selected 237 maternal-infant pairs for a validation study. The diagnoses of malformations were compared between the two data sources. While medical records may not necessarily be error-free, the diagnoses of malformations from our clinical databases and medical records were quite consistent. Compared with medical records, the sensitivity and specificity for diagnoses of malformations recorded in the Kaiser Permanente Northern California clinical databases were $100 \%$ and $94 \%$ respectively for any malformations, and $98 \%$ and $99 \%$ respectively for diagnoses of congenital heart defects.

\section{Ascertainment of potential confounders and effect modifiers}

In addition to the underlying indications for use of antihypertensive drugs, pre-existing diabetes before the index pregnancy and being overweight during pregnancy were probably the two most important potential confounders or effect modifiers. Women in the study cohorts with a pre-existing diagnosis of diabetes were identified through linkage to the Kaiser Permanente Northern California diabetes registry. Kaiser Permanente Northern California has a well established diabetes registry with over 200000 members that ascertains, verifies, and follows up all Kaiser Permanente Northern California members with a diagnosis of diabetes (type 1, type 2, or previous gestational diabetes). The registry has been estimated to be $98 \%$ sensitive for diagnosed diabetes (compared with self reports derived from a survey in 2000)..$^{10}$ This registry allowed us to identify accurately women with a diagnosis of pre-existing diabetes and provided an opportunity to examine the potential interaction between use of ACE inhibitors during pregnancy and pre-existing diabetes. 
In the current study population, about $70 \%$ of pregnant women had information on body weight during the second trimester (around 16-20 weeks of gestation) when they took part in prenatal screening. Information on height was not available for most subjects, however, so body mass index could not be calculated. We used the 90th centile of body weight in the second trimester (207 pounds $(94 \mathrm{~kg})$ ) in our study population as the cut-off point for overweight.

From the Kaiser Permanente Northern California databases, we also had information on maternal age, ethnicity, and parity for the pregnant women included. Through linkage to the California State birth certificate data, we were able to obtain information on some additional potential confounders including maternal education levels and smoking during pregnancy. However, because the California State birth certificate data were available only up to 2006, we had missing data for those two potential confounders for the births in 2007-8.

\section{Analyses}

We used logistic regression to control for confounders (maternal age, ethnicity, parity, pre-existing diabetes, and overweight) while examining the effect of maternal exposure to ACE inhibitors and other antihypertensive drugs during pregnancy on the risk of malformations in offspring. Because the rate of malformation is relatively low, odds ratios and associated $95 \%$ confidence intervals were used as approximation for relative risk to measure the strength of the association and stability of the estimates.

In addition to establishing a "hypertension only" cohort to control for the underlying indication for antihypertensive drugs, we extensively examined two important potential confounders, pre-existing diabetes and overweight. Both were adjusted for in the analyses and stratified for examination of potential modifying effect. Other potential confounders including maternal age, ethnicity, and parity were also controlled for throughout the analyses. Finally, for some variables that were available only from the California State birth certificate data (thus, available only for births up to 2006), we conducted separate analyses adjusting for those few variables among a subset of the study population including births up to 2006 .

\section{Results}

Overall, the study included 465754 mother-infant pairs. The prevalence of ACE inhibitor use during pregnancy was 1.6/1000 pregnancies, and use of other antihypertensive drugs was 38/1000. The prevalence of ACE inhibitor use in the first trimester only was $0.9 / 1000$ and in the second or third trimester only was $0.1 / 1000$. The prevalence of use of other antihypertensive drugs in the corresponding periods was $2.4 / 1000$ and $26.5 / 1000$ respectively.

Table $1 \Downarrow$ presents the characteristics of the four comparison cohorts. Compared with both normal controls and hypertension controls, women who used ACE inhibitors during pregnancy were noticeably older and more likely to be African-American, overweight ( $\geq 207$ pounds ( $94 \mathrm{~kg})$ ), have pre-existing diabetes, and have more live born children. On the other hand, the characteristics of those who used other antihypertensive drugs were similar to those of the normal controls and hypertension controls except that they were slightly more overweight than the normal controls but similar in weight to the hypertension controls.

\section{Maternal exposure to antihypertensive drugs and risk of major malformations in offspring}

Table $2 \Downarrow$ presents results of the association between maternal exposure to antihypertensive drugs, especially ACE inhibitors, during various periods of pregnancy and the risk of major malformations in offspring after adjustment for maternal age, ethnicity, parity, pre-existing diabetes, and overweight. For maternal exposure during the first trimester only, use of ACE inhibitors was associated with about $20 \%$ increased risk of any malformations in offspring compared with normal controls $(34 / 400(8.5 \%) v 22429 / 416218$ (5.4\%) cases), though this was not statistically significant. However, this association was not unique to use of ACE inhibitors. Use of other antihypertensive drugs showed a similar association (79/1141 $(6.9 \%)$ cases of birth defects, $22 \%$ increased risk). Presence of hypertension during pregnancy was also associated with a similarly increased risk of malformations in offspring $(2247 / 31$ $274(7.2 \%)$ cases, odds ratio 1.25 (95\% confidence interval 1.19 to 1.31 )) (table $2 \Downarrow$ ). Compared with the hypertension controls, there was no increased risk of malformation associated with use of either ACE inhibitors or other antihypertensive drugs. Analyses for use of antihypertensives during the first trimester, regardless of use in other trimesters, showed a largely similar pattern of association (58/704 (8.2\%) for ACE inhibitors, $327 / 4390(7.5 \%)$ for other antihypertensives).

\section{Maternal exposure to antihypertensive drugs and risk of congenital heart and neural tube defects in offspring}

The association with congenital heart defects (ICD-9 codes 745-747) and neural tube defects (ICD-9 codes 740-742) was further examined because these two types of malformations were reported specifically as being associated with ACE inhibitor use during the first trimester. ${ }^{5}$ The association between use of antihypertensive drugs (ACE inhibitor or otherwise) during the first trimester only and the risk of congenital heart defects in offspring showed a similar pattern of the association. Although maternal exposure to ACE inhibitors during the first trimester only was potentially associated with a $54 \%$ increased risk of congenital heart defects in offspring compared with normal controls $(15 / 381(3.9 \%) v 6232 / 400021$ (1.6\%) cases, odds ratio 1.54 ( 0.90 to 2.62$)$ ), use of other antihypertensive drugs was also associated with a $52 \%$ increased risk of congenital heart defects $(28 / 1090(2.6 \%)$ cases, odds ratio 1.52 (1.04 to 2.21)). Even the presence of hypertension itself during pregnancy was associated with a $41 \%$ increased risk of congenital heart defects $(708 / 29735(2.4 \%)$ cases, odds ratio 1.41 (1.30 to 1.53$)$ ). Therefore, compared with the hypertension controls, use of antihypertensive drugs in pregnancy was not associated with increased risk for congenital heart defects.

For neural tube defects, maternal use of either ACE inhibitors or other antihypertensives was not associated with increased risk, whereas the presence of hypertension during pregnancy itself was associated with an increased risk of neural tube defects (table $2 \Downarrow$ ). The results among those who used ACE inhibitors in the first trimester regardless of use during other trimesters showed a similar pattern of the association.

\section{Use of antihypertensive drugs during the second or third trimesters}

Although ACE inhibitor use during the second or third trimesters only showed a similar pattern of association with any malformations, congenital heart defects, or neural tube defects as that shown by use of ACE inhibitors and other 
antihypertensives during the first trimester (table $2 \Downarrow$ ), it was also associated with a noticeably higher risk of malformations. This increased fetal risk associated with ACE inhibitor use during the second or third trimester is well documented, resulting in ACE inhibitor use being classified by the US Food and Drug Administration as a category D drug (generally not recommended for use during the second or third trimester).

\section{Influence of pre-existing diabetes and overweight}

Although pre-existing diabetes and obesity were adjusted for in the analyses above, we further controlled for these factors by excluding mothers with these two conditions. Firstly, we repeated the analyses described in table 2 among those without pre-existing diabetes. After adjustment for confounders, the results in table $3 \Downarrow$ show that the pattern of the observed association between maternal exposure to antihypertensive drugs and the risk of malformations was largely similar to that observed in the entire study population (table $2 \Downarrow$ ). The similarity existed for antihypertensive use during the first trimester as well as during other periods of pregnancy, although the association between ACE inhibitor use in the first trimester only and the risk of any malformations no longer existed in this group (tables $3 \Downarrow)$.

Secondly, we conducted similar analyses among women not overweight. We used the 90th centile of body weight (207 pounds, $94 \mathrm{~kg}$ ) during the second trimester (the only available information on body weight) as the cut-off value. We observed a similar pattern of the associations as described above in relation to pre-existing diabetes, although most associations related to ACE inhibitor use in the first trimester only were not statistically significant because of the small sample sizes in each stratum ( $<90$ th centile, $\geq 90$ th centile, and unknown) (data not shown).

\section{Use of antihypertensive drugs without underlying hypertension}

Because some antihypertensive drugs can be used for conditions other than hypertension, we further split the users of antihypertensive drugs into two categories - with and without a diagnosis of hypertension. To reduce the influence of pre-existing diabetes, we conducted the analysis among those without pre-existing diabetes. Overall, the pattern of the association remained the same: use of both ACE inhibitors and of other antihypertensives as well as hypertension itself increased the risk of malformations (table $4 \Downarrow$ ). The association was not uniquely related to ACE inhibitor use. In addition, there was no increased risk associated with use of antihypertensive drugs (ACE inhibitors or otherwise) compared with hypertension controls. Because of the reduced sample size from refinement in individual categories, many estimates were not statistically significant or could not be obtained.

\section{Additional analyses}

To examine whether there were other potential confounders in addition to those adjusted for in the models, we conducted further analyses after adjustment for maternal education level, smoking status, and complications during pregnancy in a subset of births up to 2006 for which information on those variables was available from the California State birth certificate data. The additional adjustment for those variables did not change the observed associations (data not shown).

We also conducted additional analyses to examine any potential dose-response relation. No such relation was observed with the duration of use of either ACE inhibitors or other antihypertensive drugs. In addition, restricting malformations to those diagnosed before the age of 1 year did not change the results.

\section{Discussion}

\section{Comparison with previous studies}

ACE inhibitor use during the second or third trimesters of pregnancy is known to be associated with fetal toxicity. The US Food and Drug Administration classifies ACE inhibitor use during second and third trimesters as a category D drug (known fetal risk). The potential teratogenic effect of ACE inhibitors during the first trimester was raised in a report of increased risk of malformations after maternal exposure to ACE inhibitors in the first trimester in the Tennessee Medicaid population. ${ }^{5}$ This observed association was specifically related to ACE inhibitor use and was not present for use of other antihypertensive drugs in the study. ${ }^{5}$

Our study was based on a much larger and more ethnically diverse population with well established clinical, pharmacy, and registry databases. Although we observed an increased risk of malformations associated with ACE inhibitor use in the first trimester compared with normal controls, this association was not unique to ACE inhibitors. Use of other antihypertensive drugs in the first trimester showed a similarly increased risk of malformations in both the overall population and in those women without pre-existing diabetes. Therefore, our results do not support the argument that maternal use of ACE inhibitors in the first trimester significantly increases the risk of fetal malformations more than any other antihypertensive drug. Our findings are consistent with those from two other studies which showed that use of ACE inhibitors and of other antihypertensives were associated with similarly increased risk of malformations. ${ }^{67}$

In addition, we observed that an underlying diagnosis of hypertension was also associated with an increased risk of malformations in offspring (tables $2 \Downarrow$ and $3 \Downarrow$ ). This raises the question whether it was the underlying hypertension or the use of antihypertensives that led to the increased risks of malformation in offspring. Compared with the hypertension controls in our study, use of antihypertensives (including ACE inhibitors) in the first trimester showed no association with increased risk of malformations. Most estimates of odds ratio were around 1. For example, the risks of congenital heart defects associated with maternal use of ACE inhibitors and of other antihypertensives in the first trimester only were 1.14 (0.65 to 1.98 ) and $1.12(0.76$ to 1.64 ) respectively (table $2 \Downarrow$ ).

Although our study did not confirm the reported unique association between maternal use of ACE inhibitors in the first trimester and increased risk of malformations, ${ }^{5}$ we did observe a stronger association between maternal exposure to ACE inhibitors during the second or third trimesters and the risk of malformations than exposure to other antihypertensive drugs. This fetal risk associated with maternal use of ACE inhibitors during the later part of pregnancy is well known. Our confirmation of this established association in the present study provides support for our findings of the association with ACE inhibitor use in the first trimester.

Many reasons might account for the different findings in our study and those of Cooper et al. ${ }^{5}$ Firstly, the study populations were quite different. Our study population was more than 15 times larger than that in the study of Cooper et al. ${ }^{5}$ In addition, our study cohorts (ACE inhibitor users, users of other antihypertensives, hypertension controls, and normal controls) 
came from the same pool of members of Kaiser Permanente Northern California, ensuring comparability among them. The earlier study population was based on the Tennessee Medicaid population, ${ }^{5}$ whereas ours represented the underlying population in the service areas of the Northern California region with a different the ethnic composition-about $48 \%$ African-Americans in the earlier study compared with $10 \%$ in our study.

\section{Strengths of the study}

By identifying and establishing a cohort of pregnant women who had hypertension but did not use antihypertensive drugs, we were able to examine and disentangle the pharmacological effect of antihypertensive drugs from the effect of underlying hypertension. Our results show that the underlying hypertension is more likely to be associated with increased risk of malformations in offspring than antihypertensive drug use in the first trimester. While hypertension in pregnancy has been associated with some adverse pregnancy outcomes, including preterm delivery and fetal growth retardation, its relation with the risk of birth defects is unclear. ${ }^{11}$ In addition, we were able to separate users of antihypertensive drugs into two groups: those with a diagnosis of hypertension and those without the diagnosis. These separate cohorts allowed further disentanglement of the effect of drugs from underlying indications for the drugs.

Another strength of our study is our detailed analyses of antihypertensive drug exposure in various periods during pregnancy (in the first trimester only, any exposure in the first trimester, and exposure in the second or third trimesters only). Such analyses allowed us to confirm the presence of the known fetal toxicity due to ACE inhibitor use during the second or third trimester, providing confidence in our overall findings. However, the small number of women who used ACE inhibitors in the second or third trimester made the estimates relatively unstable.

\section{Limitations of the study}

As with any pharmacoepidemiological study using automated databases, assumptions had to be made. Although dispensary information from the Kaiser Permanente Northern California pharmacy data was accurate, women's compliance in taking the prescribed drugs is unknown. Given that antihypertensive drugs are for serious underlying conditions, compliance was expected to be high. Any non-compliance should be similar between ACE inhibitor users and users of other antihypertensive drugs, thus not affecting the observation that use of ACE inhibitors and of other antihypertensives in the first trimester had similar risk profiles in relation to malformations in offspring.

Another potential limitation of our reliance on pharmacy databases is the inability to identify women who obtained prescriptions from pharmacies outside Kaiser Permanente Northern California. However, members of Kaiser Permanente Northern California had substantial incentives to obtain drugs from Kaiser Permanente Northern California's own pharmacies in terms of the benefit of drug coverage with low co-payment. In addition, given the ACE inhibitor use in the first trimester only was rare among the pregnant women (0.9/1000), any potential impact from women who were misclassified as non-users (because of their obtaining ACE inhibitors from outside pharmacies) would be negligible because the percentage would be so small.

Also, we had little information on the characteristics of women with treated and untreated hypertension. Therefore, we were not able to distinguish between the potential underlying differences in the risk of having a child with birth defects and treatment effect on the risk of birth defects. Nevertheless, untreated hypertension is likely to be less severe than treated hypertension, and the risk of having a child with birth defects was quite similar between women with treated hypertension (in the first trimester) and those with untreated hypertension.

Finally, our study could not rule out the possibility that ACE inhibitor use during pregnancy might be associated with the risk of some subtle defects that we were not able to examine.

\section{Conclusions}

In this large, population based, cohort study we observed a similar elevated risk of malformations in offspring among women who, during the first trimester of pregnancy, had used either ACE inhibitors or other antihypertensive drugs or who had hypertension and did not use antihypertensives. Our finding did not confirm a previously reported unique association between use of ACE inhibitors in the first trimester and risk of malformations in offspring. Our finding suggests that it is likely the underlying hypertension, rather than use of antihypertensive drugs in the first trimester, that increases the risk of malformations in offspring.

\section{We thank Roxana Odouli for her help during preparation of the manuscript.}

Contributors: D-KL (study guarantor) was responsible for the design of the study, obtaining funding, analysis and interpretation of the data, and preparation of the manuscript. CY, VT, and JRF contributed to the preparation of datasets for analyses. $C Y$ conducted data analyses. SA contributed to obtaining funding. JRF and SA reviewed the manuscript. Funding: This project was funded under Contract No 290050033 from the Agency for Healthcare Research and Quality, US Department of Health and Human Services as part of the Developing Evidence to Inform Decisions about Effectiveness (DEcIDE) programme. The Food and Drug Administration, Office of Women's Health also contributed funds. The authors of this report are responsible for its content. Statements in the report should not be construed as endorsement by the Agency for Healthcare Research and Quality, the Food and Drug Administration, or the US Department of Health and Human Services. Competing interests: All authors have completed the Unified Competing Interest form at www.icmje.org/coi_disclosure.pdf (available on request from the corresponding author) and declare: no support from any organisation for the submitted work; no financial relationships with any organisations that might have an interest in the submitted work in the previous three years, no other relationships or activities that could appear to have influenced the submitted work.

Ethical approval: Institutional Review Boards of Kaiser Permanente in Northern California and California State Committee for the Protection of Human Subjects.

Data sharing: No additional data available.

1 Barr M Jr. Teratogen update: angiotensin-converting enzyme inhibitors. Teratology 1994;50:399-409.

2 Ratnapalan S, Koren G. Taking ACE inhibitors during pregnancy. Is it safe? Can Fam Physician 2002;48:1047-9.

3 Postmarketing surveillance for angiotensin-converting enzyme inhibitor use during the first trimester of pregnancy-United States, Canada, and Israel, 1987-1995. MMWR Morb Mortal Wkly Rep 1997:46:240-2.

4 Friedman JM. ACE inhibitors and congenital anomalies. N Engl J Med 2006;354:2498-500.

5 Cooper WO, Hernandez-Diaz S, Arbogast PG, Dudley JA, Dyer S, Gideon PS, et al. Major congenital malformations after first-trimester exposure to ACE inhibitors. N Engl J Med 2006;354:2443-51

6 Caton AR, Bell EM, Druschel CM, Werler MM, Lin AE, Browne ML, et al. Antihypertensive medication use during pregnancy and the risk of cardiovascular malformations. Hypertension 2009;54:63-70.

7 Lennestal R, Otterblad OP, Kallen B. Maternal use of antihypertensive drugs in early pregnancy and delivery outcome, notably the presence of congenital heart defects in the infants. Eur J Clin Pharmacol 2009;65:615-25. 


\section{What is already known on this topic}

Use of ACE inhibitors during the first trimester of pregnancy was reported to increase the risk of birth defects whereas use of other antihypertensive drugs was not

\section{What this study adds}

This much larger study with a more ethnically diverse population did not confirm the reported finding

The similar elevated risk of malformations in offspring of women who used any antihypertensive drugs in the first trimester and women who had untreated hypertension suggests it is likely the underlying condition of hypertension that increases the risk of birth defects

8 Selby JV, Karter AJ, Ackerson LM, Ferrara A, Liu J. Developing a prediction rule from automated clinical databases to identify high-risk patients in a large population with diabetes. Diabetes Care 2001;24:1547-55.

9 Kroner BA, Billups SJ, Garrison KM, Lyman AE, Delate T. Actual versus projected cost avoidance for clinical pharmacy specialist-initiated medication conversions in a primary care setting in an integrated health system. J Manag Care Pharm 2008;14:155-63.

10 Ferrara A, Quesenberry CP, Karter AJ, Njoroge CW, Jacobson AS, Selby JV. Current use of unopposed estrogen and estrogen plus progestin and the risk of acute myocardial infarction among women with diabetes: the Northern California Kaiser Permanente Diabetes Registry, 1995-1998. Circulation 2003;107:43-8.

11 Banhidy F, Acs N, Puho EH, Czeizel AE. Chronic hypertension with related drug treatment of pregnant women and congenital abnormalities in their offspring: a population-based study. Hypertens Res 2011;34:257-63.

\section{Accepted: 05 September 2011}

\section{Cite this as: BMJ 2011;343:d5931}

This is an open-access article distributed under the terms of the Creative Commons Attribution Non-commercial License, which permits use, distribution, and reproduction in any medium, provided the original work is properly cited, the use is non commercial and is otherwise in compliance with the license. See: http://creativecommons.org/licenses/by$\mathrm{nc} / 2.0 /$ and http://creativecommons.org/licenses/by-nc/2.0/legalcode. 


\section{Tables}

Table 1| Distribution of characteristics by use of antihypertensive medications during pregnancy among 465754 women who delivered live offspring in the Kaiser Permanente Northern California region during 1995-2008. Values are numbers (percentages) of subjects

\begin{tabular}{|c|c|c|c|c|}
\hline Maternal characteristics & Normal controls $(n=416218)^{*}$ & $\begin{array}{l}\text { Hypertension controls } \\
\qquad(n=31274) \dagger\end{array}$ & $\begin{array}{l}\text { Other antihypertensive use } \\
\qquad(n=17 \text { 507)‡ }\end{array}$ & ACE inhibitor use $(n=755) \S$ \\
\hline \multicolumn{5}{|l|}{ Age at delivery (years): } \\
\hline$<20$ & $27839(6.7)$ & $2019(6.5)$ & $899(5.1)$ & $7(0.9)$ \\
\hline $20-<25$ & $73186(17.6)$ & $5210(16.7)$ & $2340(13.4)$ & $44(5.8)$ \\
\hline $25-<30$ & $119109(28.6)$ & $8124(26.0)$ & $4289(24.5)$ & $102(13.5)$ \\
\hline $30-<35$ & $118237(28.4)$ & $8648(27.7)$ & $5113(29.2)$ & $210(27.8)$ \\
\hline $35-<40$ & $62812(15.1)$ & $5554(17.8)$ & $3647(20.8)$ & $253(33.5)$ \\
\hline$\geq 40$ & $14523(3.5)$ & $1710(5.5)$ & $1204(6.9)$ & $138(18.3)$ \\
\hline Unknown & $512(0.1)$ & $9(0.03)$ & $15(0.1)$ & $1(0.1)$ \\
\hline \multicolumn{5}{|l|}{ Ethnicity: } \\
\hline White & $185778(44.6)$ & $15286(48.9)$ & $8139(46.5)$ & $264(35.0)$ \\
\hline African American & $34988(8.4)$ & 3471 (11.1) & $2473(14.1)$ & $150(19.9)$ \\
\hline Hispanic & 89377 (21.5) & 6179 (19.8) & 2833 (16.2) & $130(17.2)$ \\
\hline Asian & $84594(20.3)$ & $5143(16.4)$ & 3286 (18.8) & $159(21.1)$ \\
\hline Other & $3477(0.8)$ & $313(1.0)$ & $195(1.1)$ & $8(1.1)$ \\
\hline Unknown & $18004(4.3)$ & $882(2.8)$ & $581(3.3)$ & $44(5.8)$ \\
\hline \multicolumn{5}{|l|}{ Parity (prior live births): } \\
\hline 0 & $161182(38.7)$ & $15642(50.0)$ & $6319(36.1)$ & $202(26.8)$ \\
\hline 1 & 135153 (32.5) & $8560(27.4)$ & $5373(30.7)$ & $191(25.3)$ \\
\hline 2 & $65195(15.7)$ & $3842(12.3)$ & $3046(17.4)$ & $167(22.1)$ \\
\hline$\geq 3$ & $35375(8.5)$ & $2227(7.1)$ & $1952(11.2)$ & $134(17.8)$ \\
\hline Unknown & $19313(4.6)$ & $1003(3.2)$ & $817(4.7)$ & $61(8.1)$ \\
\hline \multicolumn{5}{|l|}{ Overweight or obesity $\uparrow:$} \\
\hline No & 263013 (63.2) & $17377(55.6)$ & $10220(58.4)$ & 326 (43.2) \\
\hline Yes & $25723(6.2)$ & $5736(18.3)$ & $2229(12.7)$ & $212(28.1)$ \\
\hline Unknown & $127482(30.6)$ & $8161(26.1)$ & $5058(28.9)$ & $217(28.7)$ \\
\hline \multicolumn{5}{|l|}{ Pre-existing diabetes: } \\
\hline Yes & $1651(0.4)$ & $689(2.2)$ & $470(2.7)$ & $289(38.3)$ \\
\hline No & 414567 (99.6) & $30585(97.8)$ & 17037 (97.3) & $466(61.7)$ \\
\hline
\end{tabular}

${ }^{*}$ No use of antihypertensives during pregnancy and no diagnosis of hypertension. †No use of antihypertensives during pregnancy but with a diagnosis of hypertension. fUse of antihypertensives other than ACE inhibitors during pregnancy. $\S$ Use of ACE inhibitors during pregnancy.

ๆ90th centile of body weight in the second trimester (207 pounds, $94 \mathrm{~kg}$ ) used as cut-off point for overweight or obesity. 
Table 2| Risk of major birth defects in offspring of 465754 women who delivered in the Kaiser Permanente Northern California region during 1995-2008 by maternal use of antihypertensive medications during pregnancy

\begin{tabular}{|c|c|c|c|c|}
\hline & \multirow{2}{*}{$\begin{array}{l}\text { No }(\%) \text { of mother-infant } \\
\text { pairs }\end{array}$} & \multicolumn{3}{|c|}{ Odds ratio $(95 \% \mathrm{Cl})$} \\
\hline & & Crude & & \\
\hline \multicolumn{5}{|l|}{ Any birth defect } \\
\hline Normal controls $\dagger$ & 22 429/416 218 (5.4) & Reference & Reference & - \\
\hline$\underline{\text { Hypertension controls } \ddagger}$ & $2247 / 31274(7.2)$ & $1.36(1.30$ to 1.42$)$ & 1.25 (1.19 to 1.31$)$ & Reference \\
\hline \multicolumn{5}{|l|}{ Antihypertensive use: } \\
\hline \multicolumn{5}{|l|}{ Only in 1st trimester: } \\
\hline Other antihypertensives§ & 79/1141 (6.9) & $1.31(1.04$ to 1.64$)$ & 1.22 (0.97 to 1.54$)$ & 1.00 (0.79 to 1.27$)$ \\
\hline ACE inhibitors & $34 / 400(8.5)$ & $1.63(1.15$ to 2.32$)$ & 1.20 (0.84 to 1.72$)$ & 0.97 (0.67 to 1.41$)$ \\
\hline \multicolumn{5}{|l|}{ Any in 1 st trimester ${ }^{* \star}$} \\
\hline Other antihypertensives§ & $327 / 4390(7.5)$ & $1.41(1.26$ to 1.58$)$ & 1.29 (1.15 to 1.45$)$ & 1.07 (0.94 to 1.21$)$ \\
\hline 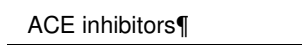 & $58 / 704(8.2)$ & 1.58 (1.21 to 2.06$)$ & 1.15 (0.87 to 1.52$)$ & 0.97 (0.73 to 1.30$)$ \\
\hline \multicolumn{5}{|l|}{ Only in 2nd or 3rd trimesters: } \\
\hline Other antihypertensives§ & 1334/13 $117(10.2)$ & 1.99 (1.88 to 2.11$)$ & 1.94 (1.83 to 2.05$)$ & 1.53 (1.43 to 1.65$)$ \\
\hline ACE inhibitors & $7 / 51(13.7)$ & $2.80(1.26$ to 6.21$)$ & 2.34 (1.05 to 5.22$)$ & 1.88 (0.84 to 4.20$)$ \\
\hline \multicolumn{5}{|l|}{ Congenital heart defectst† } \\
\hline Normal controls $\dagger$ & $6232 / 400021(1.6)$ & Reference & Reference & - \\
\hline Hypertension controls $\ddagger$ & $708 / 29735(2.4)$ & 1.54 (1.43 to 1.67$)$ & $1.41(1.30$ to 1.53$)$ & Reference \\
\hline \multicolumn{5}{|l|}{ Antihypertensive use: } \\
\hline \multicolumn{5}{|l|}{ Only in 1st trimester: } \\
\hline Other antihypertensives§ & 28/1090 (2.6) & $1.67(1.14$ to 2.43$)$ & 1.52 (1.04 to 2.21$)$ & 1.12 (0.76 to 1.64$)$ \\
\hline 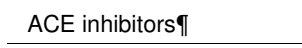 & 15/381 (3.9) & 2.59 (1.54 to 4.34$)$ & 1.54 (0.90 to 2.62$)$ & 1.14 (0.65 to 1.98$)$ \\
\hline \multicolumn{5}{|l|}{ Any in 1st trimester** } \\
\hline Other antihypertensives§ & $123 / 4186(2.9)$ & 1.91 (1.59 to 2.29 ) & 1.67 (1.39 to 2.01$)$ & 1.24 (1.02 to 1.52$)$ \\
\hline ACE inhibitors & $24 / 670(3.6)$ & 2.35 (1.57 to 3.54$)$ & 1.39 (0.91 to 2.13$)$ & $1.10(0.70$ to 1.71$)$ \\
\hline \multicolumn{5}{|l|}{ Only in 2nd or 3rd trimesters: } \\
\hline Other antihypertensives§ & $566 / 12349(4.6)$ & 3.04 (2.78 to 3.32 ) & 2.90 (2.65 to 3.16$)$ & 2.02 (1.81 to 2.27 ) \\
\hline ACE inhibitors & $4 / 48(8.3)$ & $5.75(2.06$ to 15.99$)$ & $4.28(1.53$ to 11.97$)$ & 3.04 (1.08 to 8.56$)$ \\
\hline \multicolumn{5}{|l|}{ Neural tube defects $† \dagger$} \\
\hline Normal controls $\dagger$ & $2447 / 396236(0.6)$ & Reference & Reference & - \\
\hline Hypertension controls $\ddagger$ & $281 / 29308(1.0)$ & $1.56(1.38$ to 1.76$)$ & $1.43(1.26$ to 1.62$)$ & Reference \\
\hline \multicolumn{5}{|l|}{ Antihypertensive use: } \\
\hline \multicolumn{5}{|l|}{ Only in 1st trimester: } \\
\hline Other antihypertensives§ & 9/1071 (0.8) & 1.36 (0.71 to 2.63$)$ & 1.26 (0.65 to 2.44$)$ & 0.90 (0.46 to 1.76$)$ \\
\hline ACE inhibitors & $2 / 368(0.5)$ & $0.88(0.22$ to 3.53$)$ & 0.59 (0.14 to 2.38$)$ & 0.44 (0.11 to 1.84$)$ \\
\hline \multicolumn{5}{|l|}{ Any in 1 st trimester ${ }^{\star *}$} \\
\hline Other antihypertensives $\S$ & $41 / 4104(1.0)$ & 1.62 (1.19 to 2.22$)$ & $1.46(1.07$ to 2.00$)$ & 1.07 (0.76 to 1.50$)$ \\
\hline ACE inhibitors & $6 / 652(0.9)$ & 1.50 (0.67 to 3.34$)$ & $1.03(0.45$ to 2.36$)$ & 0.86 (0.37 to 2.03$)$ \\
\hline \multicolumn{5}{|l|}{ Only in 2nd or 3rd trimesters: } \\
\hline Other antihypertensives§ & $193 / 11976(1.6)$ & 2.64 (2.27 to 3.06$)$ & 2.51 (2.16 to 2.91$)$ & 1.71 (1.41 to 2.06$)$ \\
\hline ACE inhibitors & $2 / 46(4.3)$ & 7.33 (1.78 to 30.21$)$ & $5.39(1.30$ to 22.40$)$ & 3.79 (0.90 to 15.87$)$ \\
\hline
\end{tabular}

*Adjusted for pre-existing diabetes, maternal age, ethnicity, parity, and maternal weight, and with different reference categories (normal controls or hypertension controls).

†No use of antihypertensives during pregnancy and no diagnosis of hypertension.

$\ddagger$ No use of antihypertensives during pregnancy but with a diagnosis of hypertension.

$\S$ Use of antihypertensives other than ACE inhibitors during pregnancy.

qUse of ACE inhibitors during pregnancy.

${ }^{* *}$ Any use of antihypertensives in first trimester regardless of use in other trimesters.

t†Excluding cases of other birth defects from the denominator. 
Table 3| Risk of major birth defects in offspring of 462655 women without pre-existing diabetes who delivered in the Kaiser Permanente Northern California region during 1995-2008 by maternal use of antihypertensive medications during pregnancy

\begin{tabular}{|c|c|c|c|c|}
\hline & \multirow{2}{*}{$\begin{array}{l}\text { No }(\%) \text { of mother-infant } \\
\text { pairs }\end{array}$} & \multicolumn{3}{|c|}{ Odds ratio $(95 \% \mathrm{Cl})$} \\
\hline & & Crude & & \\
\hline \multicolumn{5}{|l|}{ Any birth defect } \\
\hline Normal controls $\dagger$ & 22 277/414 567 (5.4) & Reference & Reference & - \\
\hline$\underline{\text { Hypertension controls } \ddagger}$ & $2162 / 30585(7.1)$ & $1.34(1.28$ to 1.40$)$ & 1.25 (1.19 to 1.31$)$ & Reference \\
\hline \multicolumn{5}{|l|}{ Antihypertensive use: } \\
\hline \multicolumn{5}{|l|}{ Only in 1st trimester: } \\
\hline Other antihypertensives§ & $74 / 1111(6.7)$ & 1.26 (0.99 to 1.59$)$ & $1.20(0.94$ to 1.52$)$ & 0.98 (0.77 to 1.25$)$ \\
\hline ACE inhibitors & $15 / 248(6.1)$ & $1.13(0.67$ to 1.91$)$ & 1.07 (0.64 to 1.81$)$ & 0.88 (0.52 to 1.50$)$ \\
\hline \multicolumn{5}{|l|}{ Any in 1 st trimester ${ }^{* \star}$} \\
\hline Other antihypertensives§ & $308 / 4198(7.3)$ & $1.40(1.24$ to 1.57$)$ & 1.31 (1.16 to 1.47$)$ & 1.08 (0.95 to 1.23 ) \\
\hline 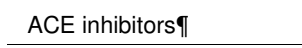 & $25 / 424(5.9)$ & $1.10(0.74$ to 1.65$)$ & $1.05(0.70$ to 1.57$)$ & 0.89 (0.59 to 1.34$)$ \\
\hline \multicolumn{5}{|l|}{ Only in 2nd or 3rd trimesters: } \\
\hline Other antihypertensives§ & 1299/12 $839(10.1)$ & $1.98(1.87$ to 2.10$)$ & 1.95 (1.84 to 2.07$)$ & 1.55 (1.44 to 1.67$)$ \\
\hline ACE inhibitors & 6/42 (14.3) & 2.94 (1.34 to 6.98$)$ & 2.78 (1.17 to 6.61$)$ & 2.22 (0.93 to 5.28 ) \\
\hline \multicolumn{5}{|l|}{ Congenital heart defectst† } \\
\hline Normal controls $\dagger$ & $6170 / 398460(1.6)$ & Reference & Reference & - \\
\hline Hypertension controls $\ddagger$ & 669/29 $092(2.3)$ & 1.49 (1.38 to 1.62$)$ & 1.40 (1.29 to 1.52$)$ & Reference \\
\hline \multicolumn{5}{|l|}{ Antihypertensive use: } \\
\hline \multicolumn{5}{|l|}{ Only in 1st trimester: } \\
\hline Other antihypertensives§ & $26 / 1063(2.5)$ & $1.59(1.08$ to 2.35$)$ & 1.50 (1.01 to 2.21$)$ & $1.10(0.74$ to 1.64$)$ \\
\hline ACE inhibitors & 8/241 (3.3) & 2.19 (1.08 to 4.42$)$ & 1.98 (0.98 to 4.02$)$ & $1.47(0.72$ to 3.00$)$ \\
\hline \multicolumn{5}{|l|}{ Any in 1st trimester** } \\
\hline Other antihypertensives§ & $116 / 4006(2.9)$ & 1.89 (1.57 to 2.29$)$ & 1.75 (1.45 to 2.11$)$ & 1.29 (1.05 to 1.59$)$ \\
\hline ACE inhibitors & $12 / 411(2.9)$ & 1.91 (1.08 to 3.40$)$ & 1.73 (0.97 to 3.07$)$ & 1.31 (0.73 to 2.35 ) \\
\hline \multicolumn{5}{|l|}{ Only in 2nd or 3rd trimesters: } \\
\hline Other antihypertensives§ & $549 / 12089(4.5)$ & 3.03 (2.77 to 3.31$)$ & 2.95 (2.70 to 3.23$)$ & 2.08 (1.85 to 2.34 ) \\
\hline ACE inhibitors & $4 / 40(10.0)$ & 7.06 (2.51 to 19.85$)$ & $6.31(2.24$ to 17.76$)$ & 4.43 (1.57 to 12.52$)$ \\
\hline \multicolumn{5}{|l|}{ Neural tube defects $† \dagger$} \\
\hline Normal controls $\dagger$ & $2425 / 394715(0.6)$ & Reference & Reference & - \\
\hline Hypertension controls $\ddagger$ & $268 / 28691(0.9)$ & $1.53(1.34$ to 1.73$)$ & $1.43(1.26$ to 1.62$)$ & Reference \\
\hline \multicolumn{5}{|l|}{ Antihypertensive use: } \\
\hline \multicolumn{5}{|l|}{ Only in 1st trimester: } \\
\hline Other antihypertensives§ & $9 / 1046(0.9)$ & $1.40(0.73$ to 2.71$)$ & 1.33 (0.69 to 2.57$)$ & 0.94 (0.48 to 1.85$)$ \\
\hline ACE inhibitors & $2 / 235(0.9)$ & $1.39(0.35$ to 5.59$)$ & $1.29(0.32$ to 5.19$)$ & 0.93 (0.23 to 3.80$)$ \\
\hline \multicolumn{5}{|l|}{ Any in 1st trimester** } \\
\hline Other antihypertensives $\S$ & $40 / 3930(1.0)$ & $1.66(1.22$ to 2.28$)$ & 1.55 (1.13 to 2.13$)$ & 1.12 (0.80 to 1.58$)$ \\
\hline ACE inhibitors & $6 / 405(1.5)$ & 2.44 (1.09 to 5.47$)$ & 2.24 (1.00 to 5.04$)$ & 1.65 (0.73 to 3.77$)$ \\
\hline \multicolumn{5}{|l|}{ Only in 2nd or 3rd trimesters: } \\
\hline Other antihypertensives§ & $185 / 11725(1.6)$ & 2.59 (2.23 to 3.02$)$ & 2.52 (2.17 to 2.93 ) & 1.71 (1.41 to 2.07 ) \\
\hline ACE inhibitors & $2 / 38(5.3)$ & $9.02(2.18$ to 37.40$)$ & 7.88 (1.89 to 32.79$)$ & 5.48 (1.31 to 22.99$)$ \\
\hline
\end{tabular}

*Adjusted for pre-existing diabetes, maternal age, ethnicity, parity, and maternal weight, and with different reference categories (normal controls or hypertension controls).

†No use of antihypertensives during pregnancy and no diagnosis of hypertension.

$\ddagger$ No use of antihypertensives during pregnancy but with a diagnosis of hypertension.

$\S$ Use of antihypertensives other than ACE inhibitors during pregnancy.

IUse of ACE inhibitors during pregnancy.

${ }^{* *}$ Any use of antihypertensives in first trimester regardless of use in other trimesters.

t†Excluding cases of other birth defects from the denominator. 
Table 4| Risk of major birth defects in offspring of 462655 women without pre-existing diabetes who delivered in the Kaiser Permanente Northern California region during 1995-2008 by maternal use of antihypertensive medications during the first trimester of pregnancy and an underlying diagnosis of hypertension

\begin{tabular}{|c|c|c|c|c|}
\hline & \multirow{2}{*}{$\begin{array}{l}\text { No (\%) of mother-infant } \\
\text { pairs }\end{array}$} & \multicolumn{3}{|c|}{ Odds ratio $(95 \% \mathrm{Cl})$} \\
\hline & & Crude & & \\
\hline \multicolumn{5}{|l|}{ Any birth defect } \\
\hline Normal controls $†$ & 22 277/414 567 (5.4) & Reference & Reference & - \\
\hline Hypertension controls $\ddagger$ & $2162 / 30585(7.1)$ & 1.34 (1.28 to 1.40$)$ & 1.25 (1.19 to 1.31$)$ & Reference \\
\hline \multicolumn{5}{|l|}{ Antihypertensive use: } \\
\hline \multicolumn{5}{|l|}{ Other antihypertensives§: } \\
\hline Without hypertension & $53 / 733(7.2)$ & $1.37(1.04$ to 1.82$)$ & $1.32(1.00$ to 1.75$)$ & 1.08 (0.81 to 1.43$)$ \\
\hline With hypertension & $21 / 378(5.6)$ & 1.04 (0.67 to 1.61$)$ & $0.96(0.62$ to 1.50$)$ & 0.81 (0.52 to 1.26$)$ \\
\hline \multicolumn{5}{|l|}{ ACE inhibitors $\uparrow:$} \\
\hline Without hypertension & $0 / 22(0)$ & NA & NA & NA \\
\hline With hypertension & $15 / 226(6.6)$ & 1.25 (0.74 to 2.11$)$ & 1.17 (0.69 to 1.98$)$ & 0.97 (0.57 to 1.65$)$ \\
\hline \multicolumn{5}{|c|}{ Congenital heart defects ${ }^{\star \star}$} \\
\hline Normal controls $\dagger$ & $6170 / 398460(1.6)$ & Reference & Reference & - \\
\hline Hypertension controlsł & 669/29 $092(2.3)$ & 1.49 (1.38 to 1.62$)$ & 1.40 (1.29 to 1.52$)$ & Reference \\
\hline \multicolumn{5}{|l|}{ Antihypertensive use: } \\
\hline \multicolumn{5}{|c|}{ Other antihypertensives§: } \\
\hline Without hypertension & $17 / 697(2.4)$ & 1.59 (0.98 to 2.57$)$ & 1.53 (0.95 to 2.48$)$ & 1.12 (0.68 to 1.82$)$ \\
\hline With hypertension & $9 / 366(2.5)$ & $1.60(0.83$ to 3.11$)$ & $1.43(0.74$ to 2.78$)$ & 1.08 (0.55 to 2.11$)$ \\
\hline \multicolumn{5}{|l|}{ ACE inhibitors $\uparrow:$} \\
\hline Without hypertension & $0 / 22(0)$ & NA & NA & NA \\
\hline With hypertension & $8 / 219(3.7)$ & 2.41 (1.19 to 4.89$)$ & 2.16 (1.07 to 4.39$)$ & 1.62 (0.79 to 3.31$)$ \\
\hline \multicolumn{5}{|l|}{ Neural tube defects ${ }^{\star *}$} \\
\hline Normal controls $\dagger$ & $2425 / 394715(0.6)$ & Reference & Reference & - \\
\hline Hypertension controlsł & $268 / 28691(0.9)$ & $1.53(1.34$ to 1.73$)$ & $1.43(1.26$ to 1.62$)$ & Reference \\
\hline \multicolumn{5}{|l|}{ Antihypertensive use: } \\
\hline \multicolumn{5}{|c|}{ Other antihypertensives§: } \\
\hline Without hypertension & $5 / 685(0.7)$ & 1.19 (0.49 to 2.87$)$ & 1.15 (0.48 to 2.77$)$ & 0.81 (0.33 to 1.96$)$ \\
\hline With hypertension & $4 / 361(1.1)$ & $1.81(0.68$ to 4.86$)$ & $1.66(0.62$ to 4.46$)$ & 1.21 (0.44 to 3.27$)$ \\
\hline \multicolumn{5}{|l|}{ ACE inhibitors $\uparrow:$} \\
\hline Without hypertension & $0 / 22(0)$ & NA & NA & NA \\
\hline With hypertension & $2 / 213(0.9)$ & $1.53(0.38$ to 6.18$)$ & $1.41(0.35$ to 5.69$)$ & 1.04 (0.25 to 4.22$)$ \\
\hline \multicolumn{5}{|c|}{$\begin{array}{l}\text { *Adjusted for pre-existing diabetes, maternal age, ethnicity, parity, and maternal weight, and with different reference categories (normal controls or hypertensi } \\
\text { controls). } \\
\text { †No use of antihypertensives during pregnancy and no diagnosis of hypertension. } \\
\text { †No use of antihypertensives during pregnancy but with a diagnosis of hypertension. } \\
\text { §Use of antihypertensives other than ACE inhibitors during pregnancy. } \\
\text { ףUse of ACE inhibitors during pregnancy. } \\
{ }^{*} \text { Excluding cases of other birth defects from the denominator. }\end{array}$} \\
\hline
\end{tabular}

